\title{
Effective nonlinear model for electron transport in deformable helical molecules
}

\author{
E. Díaz, A. Contreras, J. Hernández, and F. Domínguez-Adame \\ GISC, Departamento de Física de Materiales, Universidad Complutense, E-28040 Madrid, Spain
}

(Received 15 June 2018; published 21 November 2018)

\begin{abstract}
The helical conformation of electric dipoles in some chiral molecules, such as DNA and bacteriorhodopsin, induces a spin-orbit coupling that results in a sizable spin selectivity of electrons. The local deformation of the molecule about the moving electron may affect the spin dynamics due to the appearance of bright solitons with well-defined spin projection onto the molecule axis. In this work, we introduce an effective model for electron transport in a deformable helical molecular lattice that resembles the nonlinear Kronig-Penney model in the adiabatic approximation. In addition, the continuum limit of our model is achieved when the dipole-dipole distance is smaller than the spatial extent of the bright soliton, as discussed by E. Díaz et al. [N. J. Phys. 20, 043055 (2018)]. In this limit, our model reduces to an extended Davydov model. Finally, we also focus on perturbations to the bright soliton that arise naturally in the context of real helical molecules. We conclude that the continuum approximation provides excellent results in more complex scenarios.
\end{abstract}

DOI: 10.1103/PhysRevE.98.052221

\section{INTRODUCTION}

Several experiments on electron transport in DNA $[1,2]$ and bacteriorhodopsin on nonmagnetic metallic substrates [3] found unexpected high spin selectivity. Since spin-orbit coupling (SOC) is relatively weak in organic molecules, it was suggested that spin selectivity may be related to the helical conformation of the molecule [2]. A number of theoretical models have been put forward to elucidate the impact of the helical geometry on charge transport experiments [4-12]. In addition to geometry, other mechanisms play a major role, such as dephasing [13,14], the leakage of electrons from the molecule to the environment [15], the role of the bonding of the molecule to the metallic leads [16,17], or the interplay between a helicity-induced SOC and a strong dipole electric field, which is characteristic of these molecules [18]. The dipole electric field is created by the backbone of the molecule. The backbone with a helical arrangement of peptide dipoles wraps the cylindrical structure of the molecule. Therefore, an electron moving along the axis of the helical molecule interacts with the electric field created by the dipoles. This field results in an effective SOC that resembles the Rashba-type spin-orbit interaction [6].

Electron transport in molecules is always affected by the motion of the constituent ions [19]. Stochastic fluctuations of the ions of the molecule cause loss of coherence and energy dissipation. In addition, the local deformation of the molecule about the carrier gives rise to nonlinear effects that ultimately can lead to self-trapping [20]. Nevertheless, theoretical models have taken into account the effects of molecular deformation and nonlinear interactions only very recently [21-24].

In this work, we investigate electron transport subject to the fluctuations of the backbone of the peptide dipoles in helical molecules. We assume that each electric dipole vibrates independently of the others along the molecule axis. This is not a serious limitation and tangential displacements of the dipoles, reorientation or interdipole coupling could be easily taken into account if needed. In the framework of the adiabatic approximation, we will show that the Schrödinger equation for the electron reduces to the well-known nonlinear Kronig-Penney model (see Ref. [25] and references therein) under reasonable assumptions.

\section{ELECTRON SPIN HAMILTONIAN IN A RIGID HELICAL MOLECULE}

In this section we present an overview of the spin dynamics in rigid helical molecules according to the one-dimensional model introduced in Ref. [12]. A backbone of peptide dipoles often wraps the molecule, where the dipoles are aligned parallel to the molecule helix [26]. When the molecule is attached to magnetic or nonmagnetic metallic leads, as in the experiments mentioned above, it becomes charged. Charging effects will surely have an impact on the electric field distribution in the molecules and we will assume that it can still be described as originating from a helical arrangement of point dipoles. The electric dipole field reflects the helical arrangement of peptide dipoles and induces an unconventional Rashba-like SOC onto an electron moving along the helical axis.

Therefore, we consider a very long helix of radius $R$ directed along the $Z$ axis with axially oriented dipoles, as depicted in Fig. 1(a). The point dipoles are located at positions $\boldsymbol{r}_{n}^{0}=n \Delta z \widehat{\boldsymbol{e}}_{z}+R \widehat{\boldsymbol{\rho}}_{n}$ and dipole moments are assumed of the same magnitude $\boldsymbol{d}_{n}=d \widehat{\boldsymbol{e}}_{z}$. Here we have used cylindrical coordinates so that $\widehat{\boldsymbol{\rho}}_{n}=\left(\cos \varphi_{n}, \sin \varphi_{n}, 0\right)$ with $\varphi_{n}=$ $2 \pi n / N_{d}+\pi$. The pitch of the helix is $b=N_{d} \Delta z$, where $N_{d}$ is the number of dipoles per turn and $\Delta z$ is the spacing of the $z$ component of the position vector of the dipoles.

Each dipole contributes to the total electric field in the molecule as follows:

$$
\boldsymbol{E}(\boldsymbol{r})=\sum_{n}\left[3 \frac{\left(\boldsymbol{r}-\boldsymbol{r}_{n}^{0}\right) \cdot \boldsymbol{d}_{n}}{\left|\boldsymbol{r}-\boldsymbol{r}_{n}^{0}\right|^{5}}\left(\boldsymbol{r}-\boldsymbol{r}_{n}^{0}\right)-\frac{\boldsymbol{d}_{n}}{\left|\boldsymbol{r}-\boldsymbol{r}_{n}^{0}\right|^{3}}\right],
$$



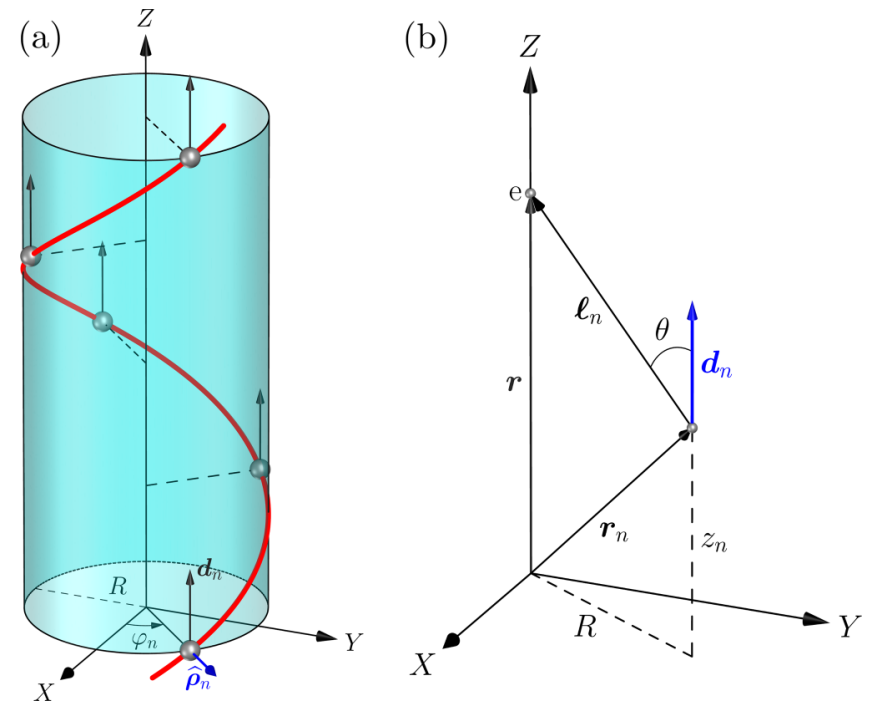

FIG. 1. (a) An electron in a given spin state is moving along the axis $Z$ of a helical arrangement of electric dipoles $\boldsymbol{d}_{n}$. The electric field created by the dipoles induces a magnetic field in the rest frame of the electron and hence influences its spin dynamics. (b) Schematic view of the various magnitudes involved in the model.

in the CGS system of units. We will restrict ourselves to electrons moving along the $Z$ axis $\left(\boldsymbol{r}=z \widehat{\boldsymbol{e}}_{z}\right)$ and neglect the motion in the transverse direction for the moment. A chiral electric potential causes a significant coupling between electron linear momentum and its spin, even for the situation in which the bare atomic SOC is small, as in most organic molecules composed by light atoms [27]. In the electron reference frame, special relativity establishes that it sees a magnetic field given as $\boldsymbol{B}=\boldsymbol{p} \times \boldsymbol{E} /\left(m c^{2}\right)$. The magnetic field lifts the spin degeneracy and ultimately yields spin selectivity through the spin-dependent electron scattering through the helical molecule attached to leads. Electron scattering can be accounted for by considering the SOC Hamiltonian arising from this mechanism. The corresponding Hamiltonian is expressed as $\mathcal{H}_{\mathrm{SO}}=\lambda \boldsymbol{\sigma} \cdot(\boldsymbol{p} \times \boldsymbol{E})$, symmetrized such that the Hamiltonian is Hermitian. Here $\lambda=e \hbar /(2 m c)^{2}$ and $\sigma$ is a vector whose components are the Pauli matrices $\sigma_{x}, \sigma_{y}$, and $\sigma_{z}$. For $\boldsymbol{p}=p_{z} \widehat{\boldsymbol{e}}_{z}$ with $p_{z}=-i \hbar \partial / \partial z$ the symmetrized SOC Hamiltonian reduces to

$$
\mathcal{H}_{\mathrm{SO}}=\frac{\lambda}{2}\left\{p_{z}\left[\begin{array}{lc}
0 & \mathcal{E}(z) \\
\mathcal{E}^{*}(z) & 0
\end{array}\right]+\left[\begin{array}{ll}
0 & \mathcal{E}(z) \\
\mathcal{E}^{*}(z) & 0
\end{array}\right] p_{z}\right\},
$$

where $\mathcal{E}(z)=-i E_{x}(z)-E_{y}(z)$. The transverse components of the dipole electric field (1) when $\boldsymbol{r}=z \widehat{\boldsymbol{e}}_{z}$ are given as

$$
\begin{aligned}
& E_{x}(z)=-3 R d \sum_{n=-\infty}^{\infty} \frac{(z-n \Delta z) \cos \varphi_{n}}{\left[R^{2}+(z-n \Delta z)^{2}\right]^{5 / 2}}, \\
& E_{y}(z)=-3 R d \sum_{n=-\infty}^{\infty} \frac{(z-n \Delta z) \sin \varphi_{n}}{\left[R^{2}+(z-n \Delta z)^{2}\right]^{5 / 2}} .
\end{aligned}
$$

Therefore, we get

$$
\mathcal{E}(z)=-i 3 R d \sum_{n=-\infty}^{\infty} \frac{(z-n \Delta z) e^{-i 2 \pi n / N_{d}}}{\left[R^{2}+(z-n \Delta z)^{2}\right]^{5 / 2}} .
$$

In most helical molecules $\Delta z \ll b$ and, as a consequence, the condition $N_{d} \gg 1$ typically holds. Under this assumption, the continuum approximation is valid (see also Sec. V A) and we can replace the sum over $n$ in Eq. (2) by an integral. Defining the dimensionless variable $u=(z-n \Delta z) / b$ and making the formal substitution $\sum_{n} \rightarrow(b / \Delta z) \int d u$ in the summation appearing in Eq. (2c) yields

$$
\begin{aligned}
& \sum_{n=-\infty}^{\infty} \frac{(z-n \Delta z) e^{-i 2 \pi(z-n \Delta z) / b}}{\left[R^{2}+(z-n \Delta z)^{2}\right]^{5 / 2}} \\
& \simeq \frac{1}{b^{3} \Delta z} \int_{-\infty}^{\infty} d u \frac{u e^{i 2 \pi u}}{\left(u^{2}+R^{2} / b^{2}\right)^{5 / 2}} .
\end{aligned}
$$

The integration is then straightforward and the result is

$$
\mathcal{E}(z)=\frac{8 \pi^{2} d}{\Delta z b^{2}} K_{1}(2 \pi R / b) e^{-i 2 \pi z / b} \equiv \mathcal{D}_{0} e^{-i 2 \pi z / b},
$$

where $K_{1}$ is the modified Bessel function of the second kind. The total Hamiltonian of the electron moving along the molecular axis subject to the SOC is then $\mathcal{H}_{L}=p_{z}^{2} / 2 m+\mathcal{H}_{\mathrm{SO}}$ whence

$$
\begin{aligned}
\mathcal{H}_{L}= & -\frac{\hbar^{2}}{2 m} \frac{\partial^{2}}{\partial z^{2}}-\hbar \lambda \mathcal{D}_{0} \\
& \times\left[\begin{array}{lc}
0 & e^{-i 2 \pi z / b}\left(i \frac{\partial}{\partial z}+\frac{\pi}{b}\right) \\
e^{i 2 \pi z / b}\left(i \frac{\partial}{\partial z}-\frac{\pi}{b}\right) & 0
\end{array}\right] .
\end{aligned}
$$

Previous models in the literature have estimated a phenomenological SOC to be of the order of $\hbar \lambda \mathcal{D}_{0}=4-$ $12 \mathrm{meV} \mathrm{nm} \mathrm{[6].} \mathrm{The} \mathrm{electrostatic} \mathrm{potential} \mathrm{due} \mathrm{to} \mathrm{the} \mathrm{helical}$ arrangement of dipoles is constant along the molecule axis and it can then be neglected. Spin dynamics in the rigid lattice obeys the Schrödinger equation associated to the linear Hamiltonian $\mathcal{H}_{L}$ and it has been thoroughly discussed in Ref. [12].

\section{ELECTRON INTERACTION WITH A SINGLE VIBRATING DIPOLE}

We assume that each electric dipole vibrates along the molecule axis independently of the others. In addition, we neglect the impact of the passing electron on the vibration frequency. As seen in the schematic view of Fig. 1(b), an electron moving along the $Z$ axis is subject to the electric potential created by a dipole located at $\boldsymbol{r}_{n}=z_{n} \widehat{\boldsymbol{e}}_{z}+R \widehat{\boldsymbol{\rho}}_{n}$. The electric potential is given as $\Phi_{n}(z)=\left(d / 4 \pi \epsilon_{0} \ell_{n}^{2}\right) \cos \theta$, where $\ell_{n}=\left|\ell_{n}\right|=\left|\boldsymbol{r}-\boldsymbol{r}_{n}\right|, \boldsymbol{r}=z \widehat{\boldsymbol{e}}_{z}$, and $\theta$ is the angle between $\boldsymbol{d}_{n}$ and $\boldsymbol{\ell}_{n}$. Now we write $z_{n}=z_{n}^{0}+u_{n}, z_{n}^{0}=n \Delta z$ being the $Z$ coordinate of the electric dipole in equilibrium (rigid molecule), and Taylor-expand the electric potential up to first order in the displacement $u_{n}$. Therefore we can write $\Phi_{n}(z) \simeq \Phi_{n}^{0}(z)+\Phi_{n}^{1}(z)$. Here $\Phi_{n}^{0}(z)$ is the electric potential that gives rise to the dipole electric field (1) after summing up the contribution of all dipoles.

The first-order correction $\Phi_{n}^{1}(z)$ takes into account the electron-dipole interaction, and it can be cast in the form

$$
\Phi_{n}^{1}(z)=-u_{n} \frac{d}{R^{3}} F\left(\frac{z-n \Delta z}{R}\right),
$$


with $F(x)=\left(1-2 x^{2}\right) /\left(1+x^{2}\right)^{5 / 2}$. The first-order correction is noticeable only when the $Z$ coordinate of the electron is close to $n \Delta z$. Consequently, we can approximate $F(x)$ by a Lorentzian since $F(x) \simeq 1-(9 / 2) x^{2} \simeq\left(1+9 x^{2} / 2\right)^{-1}$ for small $x$. In doing so, the potential energy arising from the first-order correction $V_{n}^{1}(z)=-e \Phi_{n}^{1}(z)$ is approximately given by

$$
V_{n}^{1}(z)=\widetilde{g} u_{n} \frac{\Gamma}{\pi\left[(z-n \Delta z)^{2}+\Gamma^{2}\right]} \equiv \widetilde{g} u_{n} v(z-n \Delta z),
$$

with $\widetilde{g} \equiv \sqrt{2} \pi e d / 3 R^{2}$ and $\Gamma \equiv \sqrt{2} R / 3$ is the Lorentzian half-width.

\section{DERIVATION OF THE NONLINEAR MODEL}

The derivation closely follows that of Bang et al. [28], except that the present system describes the electron dynamics in a continuous space and its spin plays a major role. In addition, we will restrict ourselves to zero temperature and neglect the interaction of the vibrating dipoles system with a thermal reservoir. Thus, the system Hamiltonian is split as $\widehat{H}=\widehat{H}_{L}+\widehat{H}_{\text {vib }}+\widehat{H}_{\text {int }}$.

The first contribution to $\widehat{H}$ is the electron Hamiltonian in the rigid molecule

$$
\widehat{H}_{L}=\sum_{\sigma, \sigma^{\prime}} \int \widehat{\psi}_{\sigma}^{\dagger}(z) \mathcal{H}_{\sigma \sigma^{\prime}} \widehat{\psi}_{\sigma^{\prime}}(z) d z,
$$

where $\widehat{\psi}_{\sigma}(z)$ is the electron field operator and $\mathcal{H}_{\sigma \sigma^{\prime}}$ denotes the elements of $\mathcal{H}_{L}$ given in Eq. (3). On the other hand, vibrations of frequency $\omega_{0}$ are treated classically according to the following Hamiltonian:

$$
\widehat{H}_{\text {vib }}=\sum_{n}\left(\frac{p_{n}^{2}}{2 M}+\frac{1}{2} M \omega_{0}^{2} u_{n}^{2}\right),
$$

where $M$ is the mass of the dipoles and $p_{n}=M \dot{u}_{n}$. Finally, the last term takes into account the electron-dipole interaction from Eq. (5) that is diagonal in the spin index

$$
\widehat{H}_{\text {int }}=\int \widehat{\psi}_{\sigma}^{\dagger}(z) \sum_{n, \sigma} \widetilde{g} u_{n} v(z-n \Delta z) \widehat{\psi}_{\sigma}(z) d z .
$$

The classical Hamiltonian is given by the trace over the electron degrees of freedom $H=\operatorname{Tr}[\widehat{\rho}(t) \widehat{H}]$, where $\widehat{\rho}(t)$ is the nonequilibrium density matrix. From the Hamilton equation $p_{n}=-\partial H / \partial u_{n}$ we are left with the equation of motion for the dipole displacements along the molecule axis

$$
\begin{aligned}
& M \ddot{u}_{n}(t)+M \omega_{0}^{2} u_{n}(t)+\widetilde{g} \sum_{\sigma} \int \rho_{\sigma \sigma}(z, z, t) v(z-n \Delta z) d z \\
& \quad=0,
\end{aligned}
$$

where the classical function is defined as [28]

$$
\rho_{\sigma \sigma^{\prime}}\left(z, z^{\prime}, t\right)=\operatorname{Tr}\left[\widehat{\rho}(t) \widehat{\psi}_{\sigma}^{\dagger}(z) \widehat{\psi}_{\sigma^{\prime}}\left(z^{\prime}\right)\right] .
$$

We now consider the quantum mechanical Liouville equation for the density matrix

$$
i \hbar \frac{d \widehat{\rho}}{d t}=[\widehat{H}, \widehat{\rho}]
$$

Inserting the Hamiltonian $\widehat{H}=\widehat{H}_{L}+\widehat{H}_{\text {vib }}+\widehat{H}_{\text {int }}$ into Eq. (8a) we obtain an equation for the classical function $\rho_{\sigma \sigma^{\prime}}\left(z, z^{\prime}, t\right)$. Assuming that it may be written as a product [28]

$$
\rho_{\sigma \sigma^{\prime}}\left(z, z^{\prime}, t\right)=\chi_{\sigma}^{*}(z, t) \chi_{\sigma^{\prime}}\left(z^{\prime}, t\right),
$$

we get the following equation for the carrier:

$$
\begin{aligned}
i \hbar \frac{\partial}{\partial t} \chi_{\sigma}(z, t)= & \sum_{\sigma^{\prime}} \mathcal{H}_{\sigma \sigma^{\prime}}(z) \chi_{\sigma^{\prime}}(z, t) \\
& +\widetilde{g} \sum_{n} v(z-n \Delta z) \chi_{\sigma}(z, t) u_{n}(t),
\end{aligned}
$$

along with the equation for the dipole displacements (7a) that can be cast in the form

$$
\begin{aligned}
& M \ddot{u}_{n}(t)+M \omega_{0}^{2} u_{n}(t) \\
& \quad+\widetilde{g} \sum_{\sigma} \int \chi_{\sigma}^{*}(z, t) \chi_{\sigma}(z, t) v(z-n \Delta z) d z=0 .
\end{aligned}
$$

Equations (9a) and (9b) constitute the starting point of our analysis of the electron dynamics in the deformable helical molecule. It should be stressed that noise and dissipation are not considered in the classical equation of motion for the dipole displacements. Therefore, our approach holds at low temperature.

\section{ADIABATIC APPROXIMATION AND EFFECTIVE MODEL}

In order to elucidate the impact of the vibration of dipoles on the electron spin dynamics, we shall introduce several reasonable assumptions. First, we can effectively decouple Eqs. (9) by assuming that the inverse of the dipole frequency $\omega_{0}^{-1}$ is much larger than any characteristic time of the electron dynamics (adiabatic approximation). This can be accomplished by setting $\ddot{u}_{n}(t) \approx 0$ in Eq. (9b) and inserting the result into Eq. (9a). After doing this, we obtain

$$
\begin{aligned}
i \hbar \frac{\partial}{\partial t} \chi_{\sigma}(z, t)= & \sum_{\sigma^{\prime}} \mathcal{H}_{\sigma \sigma^{\prime}}(z) \chi_{\sigma^{\prime}}(z, t) \\
& -x s \frac{\widetilde{g}^{2}}{M \omega_{0}^{2}} \sum_{n} v(z-n \Delta z) \chi_{\sigma}(z, t) \\
& \times \sum_{\sigma^{\prime}} \int \chi_{\sigma^{\prime}}^{*}\left(z^{\prime}, t\right) \chi_{\sigma^{\prime}}\left(z^{\prime}, t\right) v\left(z^{\prime}-n \Delta z\right) d z^{\prime} .
\end{aligned}
$$

The adiabatic approximation turns the original many-body problem into an effective one-body problem described by a nonlinear equation. Despite this simplification, Eq. (10) is still complicated due to the occurrence of the nonlocal interaction term. In the weak electron-dipole coupling limit, i.e., when $\widetilde{g}$ is small enough, the electron is weakly localized and the spatial distance over which the probability density changes noticeably is larger than the molecule radius. In such a case, the Lorentzian $v(z)$ appearing in Eq. (10) and given by Eq. (5) is a sharply peaked function in comparison to the electron probability density. We can then replace $v(z)$ by a $\delta$ function 
by formally taking the limit $\Gamma \rightarrow 0$ while keeping $\widetilde{g}$ finite. Therefore, under these premises the nonlocal interaction term becomes local and Eq. (10) reduces to

$$
\begin{aligned}
i \hbar \frac{\partial}{\partial t} \chi_{\sigma}(z, t)= & \sum_{\sigma^{\prime}} \mathcal{H}_{\sigma \sigma^{\prime}}(z) \chi_{\sigma^{\prime}}(z, t) \\
& -\frac{\widetilde{g}^{2}}{M \omega_{0}^{2}} \sum_{n} \delta(z-n \Delta z) \chi_{\sigma}(z, t) \\
& \times \sum_{\sigma^{\prime}} \chi_{\sigma^{\prime}}^{*}(n \Delta z, t) \chi_{\sigma^{\prime}}(n \Delta z, t) .
\end{aligned}
$$

We now define the dimensionless space coordinate $\xi=$ $z / b$ and time $\tau=\left(E_{b} / \hbar\right) t$, where $E_{b}=\hbar^{2} / 2 m b^{2}$, along with the dimensionless SOC parameter $\gamma=\hbar \lambda \mathcal{D}_{0} /\left(2 \pi b E_{b}\right)$. From Eqs. (3) and (11) we are led to

$$
\begin{aligned}
& i \dot{\chi}(\xi, \tau)+\left[\frac{\partial^{2}}{\partial \xi^{2}}+\widehat{M}+\frac{4 g}{N_{d}}|\chi(\xi, \tau)|^{2} \sum_{n} \delta\left(\xi-n / N_{d}\right)\right] \\
& \quad \times \chi(\xi, \tau)=0
\end{aligned}
$$

where $g=N_{d} \widetilde{g}^{2} /\left(4 M \omega_{0}^{2} b E_{b}\right)$ and $\chi(\xi, \tau)$ is a twocomponent vector whose components are $\chi_{1}(\xi, \tau)$ and $\chi_{2}(\xi, \tau)$. Here the dot indicates differentiation with respect to the dimensionless time $\tau$. For sake of brevity we have defined the matrix operator

$$
\widehat{M}=2 \pi \gamma\left[\begin{array}{lc}
0 & e^{-i 2 \pi \xi}\left(i \frac{\partial}{\partial \xi}+\pi\right) \\
e^{i 2 \pi \xi}\left(i \frac{\partial}{\partial \xi}-\pi\right) & 0
\end{array}\right] .
$$

In conclusion, Eq. (12) describes the electron dynamics in a periodic array of vibrating dipoles within the adiabatic approximation.

\section{A. Continuum approximation}

For further estimations, notice that typical values are $N_{d} \sim$ 10 dipoles per turn in DNA or $N_{d} \sim 5$ in $\alpha$ helix in the secondary structure of proteins. Thus, since $N_{d} \gg 1$, we can proceed to the continuum limit and replace the summation in Eq. (12a) by an integration,

$$
\frac{1}{N_{d}} \sum_{n} \delta\left(\xi-n / N_{d}\right) \rightarrow \int_{-\infty}^{\infty} \delta(\xi-u) d u=1 .
$$

Therefore, in the continuum limit Eq. (12a) reduces to

$$
i \dot{\chi}(\xi, \tau)+\left[\frac{\partial^{2}}{\partial \xi^{2}}+\widehat{M}+4 g|\chi(\xi, \tau)|^{2}\right] \chi(\xi, \tau)=0 .
$$

This equation supports a variety of stable solitons such as bright solitons, breathers, and rogue waves, all of them presenting well-defined spin projection onto the molecule axis. A thorough study of the soliton solutions have been presented and discussed in Refs. [21,22]. In the following section we discuss how bright solitons, similarly to the wellknown Davydov's soliton [29], are affected when Eq. (13b) is modified by the presence of different perturbations.

\section{B. Generalized nonlinear Kronig-Penney model}

The nonlinear equation (12) can be further transformed. To this end, let us define the following unitary matrix:

$$
Q(\xi)=\left[\begin{array}{cc}
\mu_{+}^{*}(\xi) \sin \varphi & -\mu_{-}(\xi) \cos \varphi \\
\mu_{-}^{*}(\xi) \cos \varphi & \mu_{+}(\xi) \sin \varphi
\end{array}\right],
$$

where $\gamma=\tan (2 \varphi)$ and

$$
\mu_{ \pm}(\xi)=\exp \left[i \pi\left(\sqrt{1+\gamma^{2}} \pm 1\right) \xi\right]
$$

We now write the solution to Eq. (12) in the following form:

$$
\chi(\xi, \tau)=\frac{1}{\sqrt{2 g}} e^{i \pi^{2} \gamma^{2} \tau} Q(\xi) \boldsymbol{\Psi}(\xi, \tau) .
$$

On making this substitution one finally gets

$$
\begin{aligned}
& i \dot{\boldsymbol{\Psi}}(\xi, \tau)+\frac{\partial^{2}}{\partial \xi^{2}} \boldsymbol{\Psi}(\xi, \tau)+\frac{2}{N_{d}} \sum_{n} \delta\left(\xi-n / N_{d}\right)|\boldsymbol{\Psi}(\xi, \tau)|^{2} \\
& \quad \times \boldsymbol{\Psi}(\xi, \tau)=0 .
\end{aligned}
$$

Notice that we can get rid of the operator $\widehat{M}$ defined in Eq. (12b) by means of the unitary transformation (14a).

We seek a particular class of solutions to Eq. (14c) of the form

$$
\boldsymbol{\Psi}(\xi, \tau)=e^{i \kappa^{2} \tau} \psi(\xi) \boldsymbol{\Theta}, \quad \boldsymbol{\Theta}=\left(\begin{array}{c}
\cos \theta \\
\sin \theta
\end{array}\right),
$$

where $\theta$ and $\kappa$ are real parameters. For the sake of concreteness we will focus our attention on solutions with $\psi(\xi)$ real as well. Inserting this ansatz into Eq. (14c) we get

$$
\psi^{\prime \prime}(\xi)-\kappa^{2} \psi(\xi)+\frac{2}{N_{d}} \sum_{n} \delta\left(\xi-n / N_{d}\right) \psi^{3}(\xi)=0 .
$$

Here the prime indicates differentiation with respect to the argument. This is nothing but the well-known nonlinear KronigPenney model, as discussed by Hennig and Tsironis [25]. It becomes apparent that our model described by Eq. (12a) can be regarded as a vector nonlinear Kronig-Penney model, where an electron in a given spin state interacts with a series of periodically spaced $\delta$ function potentials modulated by the square of the amplitude of the wave function.

\section{Generalized nonlinear Kronig-Penney model in the continuum approximation}

In the cases of interest $N_{d} \gg 1$ and substitution (13a) is a reasonable approximation. In the continuum limit, the equation of motion of the nonlinear Kronig-Penney model given by Eq. (16) reduces to the following nonlinear Schrödinger equation (NLSE):

$$
\psi^{\prime \prime}(\xi)-\kappa^{2} \psi(\xi)+2 \psi^{3}(\xi)=0 .
$$

The NLSE admits a bright soliton solution of the form

$$
\psi_{0}(\xi)=\kappa \operatorname{sech}(\kappa \xi) .
$$

We can then obtain a bright soliton solution to Eq. (13b) of the continuum model with the aid of the transformations (14b) and (15)

$$
\chi_{0}(\xi, \tau)=\frac{\kappa}{\sqrt{2 g}} \operatorname{sech}(\kappa \xi) Q(\xi) e^{i\left(\pi^{2} \gamma^{2}+\kappa^{2}\right) \tau} \boldsymbol{\Theta} .
$$


Normalization of the wave function $\chi_{0}(\xi, \tau)$ implies that $\kappa=g$.

\section{EFFECTS OF PERTURBATIONS}

Having presented the existence of bright solitons in deformable helical molecules under the particular physical conditions considered in Sec. VC, we now turn to discuss the impact of some potential perturbations that arise naturally in the context of real systems. All perturbations we consider here led to a nonlinear equation of the form

$$
\begin{aligned}
i \dot{\chi} & +\left(\frac{\partial^{2}}{\partial \xi^{2}}+\widehat{M}+4 g|\chi|^{2}\right) \chi \\
& =V_{1}(\xi) \sigma_{z} \chi+2 g\left\{\chi^{\dagger} \cdot\left[V_{2}(\xi) \mathbb{1}_{2}+V_{3}(\xi) \sigma_{z}\right] \cdot \chi\right\} \chi,
\end{aligned}
$$

where the dependence of $\chi$ on $(\xi, \tau)$ has been omitted for clarity and $\mathbb{1}_{2}$ is the $2 \times 2$ identity matrix. Here the functions $V_{i}(\xi)$ are arbitrary but small. We now perform the transformations prescribed by Eqs. (14b) and (15) to obtain

$$
\begin{aligned}
& \psi^{\prime \prime}(\xi)-\kappa^{2} \psi(\xi)+2 \psi^{3}(\xi) \\
& \quad=-V_{1}(\xi) W(\xi) \psi(\xi)+\left[V_{2}(\xi)-V_{3}(\xi) W(\xi)\right] \psi^{3}(\xi),
\end{aligned}
$$

where $W(\xi)$ is defined as

$$
\begin{aligned}
W(\xi)= & \cos (2 \theta) \cos (2 \varphi) \\
& +\sin (2 \theta) \sin (2 \varphi) \cos \left(2 \pi \sqrt{1+\gamma^{2}} \xi\right) .
\end{aligned}
$$

Equation (19) will be solved assuming that the interaction terms $V_{i}(\xi)$ do not have a strong impact on the soliton dynamics. Thus, we split the wave function as $\psi(\xi)=$ $\psi_{0}(\xi)+\psi_{1}(\xi)$, where $\psi_{0}(\xi)=g \operatorname{sech}(g \xi)$ [see Eq. (17b)] and $\psi_{1}(\xi)$ is a small correction. Furthermore, we can write $\kappa=g(1+\omega / 2)$ with $|\omega| \ll 1$. Keeping only first-order terms in Eq. (19a) we get

$$
\psi_{1}^{\prime \prime}(x)+\left[6 \operatorname{sech}^{2} x-1\right] \psi_{1}(x)=U(x),
$$

where $x=g \xi$ and

$$
\begin{aligned}
U(x)= & g \omega \operatorname{sech} x-\frac{1}{g} V_{1}(x / g) W(x / g) \operatorname{sech} x \\
& +g\left[V_{2}(x / g)-V_{3}(x / g) W(x / g)\right] \operatorname{sech}^{3} x .
\end{aligned}
$$

This inhomogeneous equation can be straightforwardly solved by means of Green's function techniques [30]. To proceed, we consider two independent solutions of the homogeneous equation $[U(x)=0]$

$$
\begin{aligned}
& \phi_{1}(x)=\sinh x \operatorname{sech}^{2} x, \\
& \phi_{2}(x)=\cosh x+3(x \sinh x-\cosh x) \operatorname{sech}^{2} x .
\end{aligned}
$$

Even solutions of Eq. (20) with boundary conditions $\psi_{1}^{\prime}(0)=$ 0 and $\lim _{x \rightarrow \infty} \psi_{1}(x)=0$ are given as

$$
\psi_{1}(x)=\int_{0}^{\infty} G(x, y) U(y) d y, \quad x \geqslant 0,
$$

where it is understood that $\psi_{1}(x)=\psi_{1}(-x)$ for $x<0$. The Green's function is written in terms of the independent solutions (21a) as follows:

$$
G(x, y)=\frac{1}{2} \begin{cases}\phi_{1}(x) \phi_{2}(y), & 0 \leqslant y \leqslant x<\infty, \\ \phi_{1}(y) \phi_{2}(x), & 0 \leqslant x \leqslant y<\infty .\end{cases}
$$

Once the perturbation potential $U(x)$ is known, the solution of the nonlinear equation (18) can be obtained up to first-order corrections. In the rest of the paper we will discuss several perturbations that arise naturally in the context of deformable helical molecules.

\section{A. Validity of the continuum approximation}

In previous sections we have demonstrated that the electron dynamics coupled to vibrating dipoles can be accurately described by a generalized nonlinear Kronig-Penney model, provided that the adiabatic approximation holds. The continuum limit of this model has been exactly solved and we have found a bright soliton solution given in Eq. (17c). In this section we discuss the validity of the replacement (13a) by noticing that the generalized nonlinear Kronig-Penney model (12a) can be written in the general form (18) with $V_{1}(\xi)=V_{3}(\xi)=0$ and $V_{2}(\xi)=2-\left(2 / N_{d}\right) \sum_{n} \delta\left(\xi-n / N_{d}\right)$, leading to

$$
\begin{aligned}
U(x)= & g \omega \operatorname{sech} x \\
& +2 g\left[1-\frac{g}{N_{d}} \sum_{n} \delta\left(x-n g / N_{d}\right)\right] \operatorname{sech}^{3} x .
\end{aligned}
$$

From Eq. (21b) we find the first-order correction as $\psi_{1}(x)=$ $(g / 2)[\omega A(x)-B(x)]$, where

$$
\begin{aligned}
& A(x)=(x \sinh x-\cosh x) \operatorname{sech}^{2} x \\
& B(x)=\operatorname{sech} x+\frac{4 g}{N_{d}} \sum_{n=1}^{\infty} G\left(x, \frac{n g}{N_{d}}\right) \operatorname{sech}^{3}\left(\frac{n g}{N_{d}}\right) .
\end{aligned}
$$

The unknown parameter $\omega$ is determined from the normalization of the wave function $\chi(\xi, \tau)$ obtained with the aid of Eqs. (14b) and (15) for $\psi(\xi)=\psi_{0}(\xi)+\psi_{1}(\xi)$. Doing so, one finds two solutions for $\omega$. In our numerics, we will take the one corresponding to the lower value of the energy of the soliton.

In Fig. 2 the effect of the discretization on the electron wave function is depicted. Left panels show the first-order correction, $\psi_{1}(\xi)$, to the total wave function, $\psi(\xi)$, due to the discretization of the lattice. For completeness we also show in the right panels the comparison between the electron wave function for the continuum $\left[\psi_{0}(\xi)\right]$ and the discrete $[\psi(\xi)=$ $\left.\psi_{0}(\xi)+\psi_{1}(\xi)\right]$ models. In all cases, the lattice discretization hardly affects the electron wave function. For instance, for the upper right panel with $N_{d}=10$, the magnitude of the perturbation remains below the $10 \%$. Indeed, by increasing the number of dipoles per turn, we recover the solution of the continuum approximation. Our results provide further support to previous studies devoted to spin-transport in organic molecules such as DNA or $\alpha$ helices, where the continuum approximation was considered $[6,12,22]$.

\section{B. Out-of-axis motion}

In the derivation of the model in Sec. IV we assumed that the charge carrier moves along the molecule axis. We 

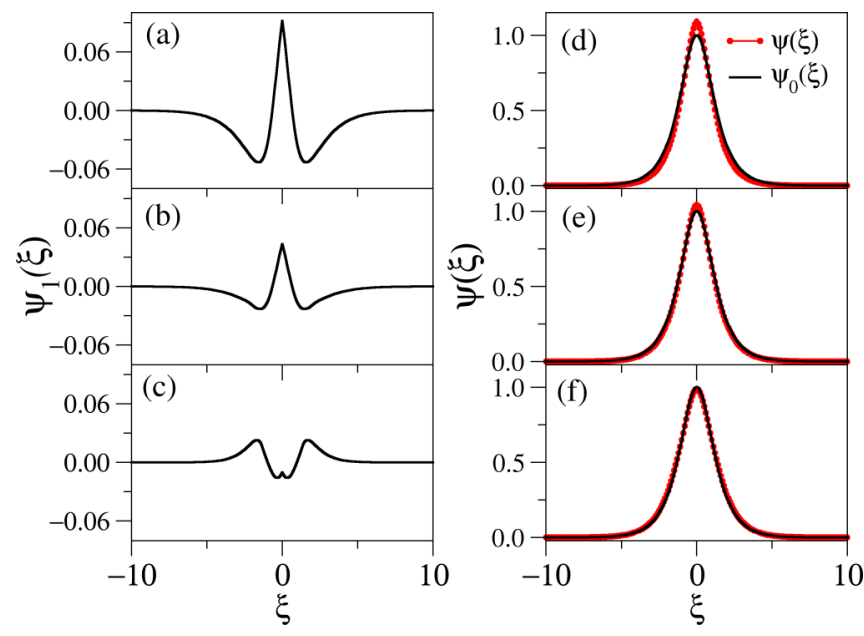

FIG. 2. Left panels show the first-order correction, $\psi_{1}(\xi)$, to the electron wave function due to the lattice discretization when the number of dipoles per turn is (a) $N_{d}=10$, (b) $N_{d}=20$ and (c) $N_{d}=$ 30. Right panels show the total wave function for the continuum model, $\psi_{0}(\xi)$, and for the discrete model, $\psi(\xi)=\psi_{0}(\xi)+\psi_{1}(\xi)$, for (d) $N_{d}=10$, (e) $N_{d}=20$ and (f) $N_{d}=30$. In all cases $g=1$.

now investigate the correctness of this approximation by letting electrons move along a helical path of radius $R^{\prime}$ and pitch $b$, where $R^{\prime}$ is of the order of the molecule radius $R$. The classical equation of the trajectory is given as $x(t)=$ $R^{\prime} \cos (2 \pi z / b), y(t)=R^{\prime} \sin (2 \pi z / b)$, and $z(t)=v t$. Here $v$ is the classical velocity, being related to the electron energy $E$ by the expression $v=\sqrt{2 E / m}$. The perpendicular (out-ofaxis) linear momentum then is $\boldsymbol{p}_{\perp}=p_{x} \widehat{\boldsymbol{e}}_{x}+p_{y} \widehat{\boldsymbol{e}}_{y}$, with

$$
\begin{aligned}
& p_{x}=-2 \pi \sqrt{2 m E}\left(\frac{R^{\prime}}{b}\right) \cos \left(2 \pi \frac{z}{b}\right), \\
& p_{y}=2 \pi \sqrt{2 m E}\left(\frac{R^{\prime}}{b}\right) \sin \left(2 \pi \frac{z}{b}\right) .
\end{aligned}
$$

The SOC Hamiltonian can be expressed as $H_{\mathrm{SOC}}=-\mu_{B} g_{0} \sigma$. $\boldsymbol{B}_{\text {eff }}$, where $\mu_{B}$ is the Bohr magneton, $g_{0}$ is the $g$ factor, and $\boldsymbol{B}_{\text {eff }}=-\boldsymbol{p} \times \boldsymbol{E} / 4 m c^{2}$. Let $\Delta \boldsymbol{B}_{\text {eff }}=-\boldsymbol{p}_{\perp} \times \boldsymbol{E} / 4 m c^{2}$ be the contribution of the perpendicular linear momentum to the effective magnetic field. The component of $\Delta \boldsymbol{B}_{\text {eff }}$ in the $X Y$ plane will not affect much the already-studied SOC [see Eq. (2a)]. Therefore, we restrict ourselves to study the component of $\Delta \boldsymbol{B}_{\text {eff }}$ along the molecule axis

$$
\begin{aligned}
\left(\Delta \boldsymbol{B}_{\mathrm{eff}}\right)_{z}= & \frac{2 \pi}{4 m c^{2}} \sqrt{2 m E}\left(\frac{R^{\prime}}{b}\right) \\
& \times\left[\sin \left(2 \pi \frac{z}{b}\right) E_{y}(z)+\cos \left(2 \pi \frac{z}{b}\right) E_{x}(z)\right]
\end{aligned}
$$

The interaction energy due to this component of the effective magnetic field resembles a Zeeman-type term of the form $H_{Z}=-\mu_{B} g_{0} \sigma_{z}\left(\Delta \boldsymbol{B}_{\text {eff }}\right)_{z}$. Since the components of the dipole electric field appearing in (23b) have already been calculated [see Eq. (1)], we finally find that

$$
H_{Z}=K E_{b} \sin (4 \pi \xi) \sigma_{z}
$$
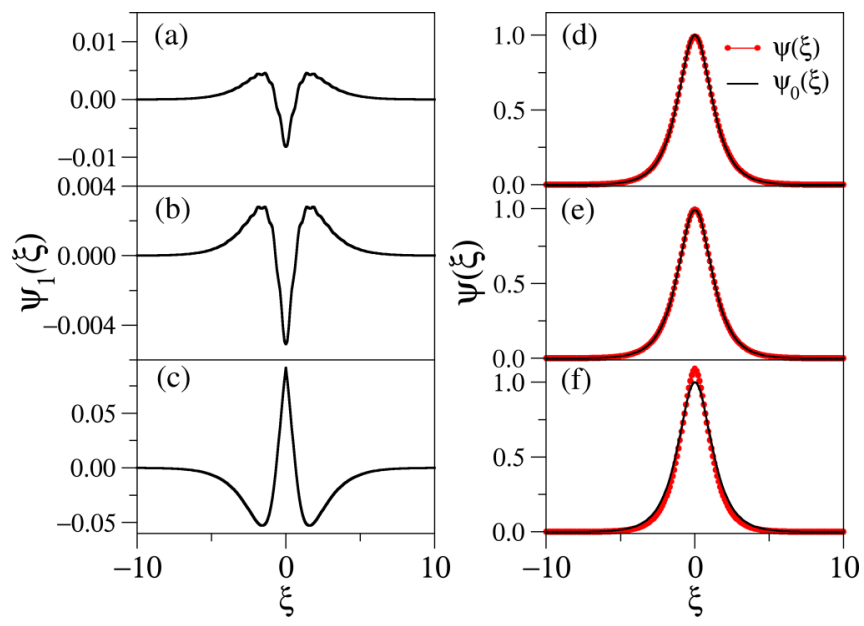

FIG. 3. Left panels show the first-order correction, $\psi_{1}(\xi)$, to the electron wave function due to the out-of-axis linear momentum for (a) $\theta=0$, (b) $\theta=\pi / 6$ and (c) $\theta=\pi / 4$. Right panels show the total wave function for the continuum model, $\psi_{0}(\xi)$, and that for an outof-axis propagation, $\psi(\xi)=\psi_{0}(\xi)+\psi_{1}(\xi)$, with $K=0.1$ and (d) $\theta=0$, (e) $\theta=\pi / 6$ and (f) $\theta=\pi / 4$. In all cases $g=1$ and $\gamma=0.1$.

where

$$
K=\frac{\pi \mathcal{D}_{0} \mu_{B} g_{s}}{m c^{2} E_{b}} \sqrt{2 m E}\left(\frac{R^{\prime}}{b}\right) .
$$

The interaction energy $H_{Z}$ is to be added to the Hamiltonian (3), leading to an equation of the form (18) with $V_{1}(\xi)=$ $K \sin (4 \pi \xi)$ and $V_{2}(\xi)=V_{3}(\xi)=0$. Therefore

$$
U(x)=\left[g \omega-\frac{K}{g} \sin \left(4 \pi \frac{x}{g}\right) W\left(\frac{x}{g}\right)\right] \operatorname{sech} x .
$$

The first-order correction is again written as $\psi_{1}(x)=$ $(g / 2)[\omega A(x)-B(x)]$, where $A(x)$ is given by (22b) and

$$
B(x)=\frac{2 K}{g^{2}} \int_{0}^{\infty} G(x, y) \sin \left(4 \pi \frac{y}{g}\right) W\left(\frac{y}{g}\right) \operatorname{sech} y d y .
$$

Figure 3 shows the effect of the perturbation $\psi_{1}(\xi)$ (see left panels) when the out-of-axis momentum component in the electron motion is taken into account. Similarly to Fig. 2, in Fig. 3 we compare the electron wave function for the unperturbed $\psi_{0}(\xi)$ and the perturbed models $\psi(\xi)=\psi_{0}(\xi)+$ $\psi_{1}(\xi)$. In this case our results depend on the angle $\theta$ by way of the function $W(y / g)$ given in Eq. (19b). This angle fixes the helicity of the electron wave function. Indeed, the effect of the perturbation is periodic with $\theta$ and, therefore, Fig. 3 only presents results for $\theta=0, \pi / 6$, and $\pi / 4$ for simplicity. Once again the effect of the perturbation is almost negligible far from the wave function peak and even in the most pronounced case $(\theta=\pi / 4)$ it remains below $10 \%$.

\section{Spin-dependent electron-dipole interaction}

According to Eq. (6c), the interaction of the electron with vibrating dipoles is diagonal in the spin index. Nevertheless, when the spin-orbit coupling is large as compared to the phonon energy, Covaci and Berciu studied the effects of the 

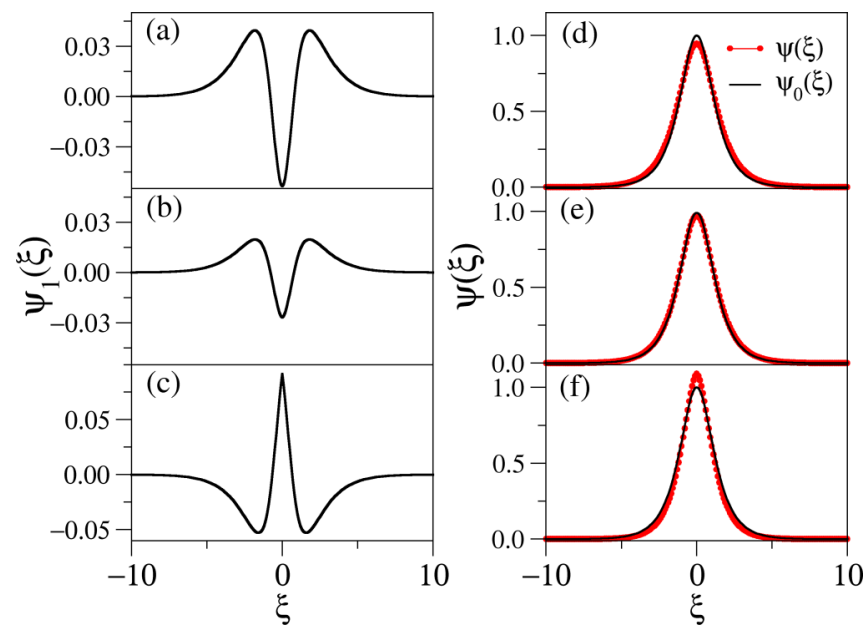

FIG. 4. Left panels show the first-order correction, $\psi_{1}(\xi)$, to the electron wave function due to the spin-dependent electron-lattice interaction $g_{ \pm}=(1 \pm \epsilon) g$ for (a) $\theta=0$, (b) $\theta=\pi / 6$ and (c) $\theta=$ $\pi / 4$. Right panels show the total wave function for the unperturbed $(\epsilon=0)$ and perturbed $(\epsilon=0.1)$ electron wave functions for (d) $\theta=0$, (e) $\theta=\pi / 6$ and (f) $\theta=\pi / 4$. In all cases $g=1$ and $\gamma=0.1$.

Rashba spin-orbit coupling on polaron formation and have found that the polaron retains only one of the spin-polarized bands in its coherent spectrum [31]. This complicated scenario can be mimicked by an effective spin-dependent electrondipole interaction in our model. To do so, we replace the spin-independent nonlinear term in Eq. (13b) as follows:

$$
g \chi^{\dagger} \cdot \chi \rightarrow \chi^{\dagger}\left[\begin{array}{ll}
g_{+} & 0 \\
0 & g_{-}
\end{array}\right] \cdot \chi=g \chi^{\dagger} \cdot \chi+\epsilon g \chi^{\dagger} \sigma_{z} \chi
$$

where $g_{ \pm}=(1 \pm \epsilon) g$. Assuming that the asymmetry parameter is small $(|\epsilon| \ll 1)$, we can apply the perturbation approach by noticing that now the NLS can be cast in the form (18) with $V_{1}(\xi)=V_{2}(\xi)=0$ and $V_{3}(\xi)=2 \epsilon$, yielding

$$
U(x)=g \omega \operatorname{sech} x-2 \epsilon \kappa W(x / \kappa) \sinh ^{3} x .
$$

The first-order correction is given as $\psi_{1}(x)=(g / 2)[\omega A(x)-$ $B(x)$ ], where $A(x)$ as in (22b) and now

$$
B(x)=4 \epsilon \int_{0}^{\infty} G(x, y) W\left(\frac{y}{g}\right) \operatorname{sech}^{3} y d y .
$$

In Fig. 4 we focus on the analysis of the effect of the spin-dependent electron-lattice interaction, such as $g_{ \pm}=(1 \pm$ $\epsilon) g$, in the molecule. Left panels show the the perturbation $\psi_{1}(\xi)$ while right panels present a comparison between the unperturbed $(\epsilon=0)$ and perturbed $(\epsilon=0.1)$ electron wave functions. As in the previous section, our results depend periodically on the angle $\theta$ so we present results for $\theta=$ $0, \pi / 6$, and $\pi / 4$. Once again the effect of the perturbation is stronger at the wave-function peak in general and for $\theta=\pi / 4$ in particular. It is worth noticing that results for $\theta=\pi / 4$ are rather similar to those found in the previous section. However, for the rest of considered values of the parameter $\theta$, the perturbation is more relevant in the case of spin-dependent electron-lattice interaction. Still, this remains below $10 \%$ as before.

\section{CONCLUSIONS}

In conclusion, we have studied the interaction of moving electrons with the vibrating dipoles of helical molecules. The electron is treated quantum mechanically and the lattice vibration is taken classically at zero temperature. The dipole electric field induces a Rashba-like SOC for electrons moving along the helical axis while the electron-lattice interaction leads to a generalized Kronig-Penney model [see Eq. (12a)] within the framework of the adiabatic approximation. Since the number of dipoles per turn is usually much larger than unity in most cases of interest $\left(N_{d} \gg 1\right)$, the continuum limit of the generalized Kronig-Penney model is a reasonable approximation and we are then led to an extended Davydov model, as discussed in Ref. [22].

The perturbation approach, introduced by Mallory and Van Gorder in Ref. [30], confirms that the discrete nature of the array of dipoles has a negligible impact on the bright solitons of the extended Davydov model. In addition, some other relevant perturbations, such as the out-of-axis motion of electrons and an effective spin-dependent electron-lattice interaction, do not alter much the bright soliton solutions of the extended Davydov model. Therefore, we can conclude that the effective nonlinear model given in Eq. (13b) provides a reasonable description of electron transport in deformable helical molecules.

\section{ACKNOWLEDGMENTS}

This work was supported by the Spanish MINECO under Grant No. MAT2016-75955. The authors are indebted to R. Gutierrez, P. G. Estévez, and P. Albares for enlightening discussions.
[1] B. Göhler, V. Hamelbeck, T. Z. Markus, M. Kettner, G. F. Hanne, Z. Vager, R. Naaman, and H. Zacharias, Science 331, 894 (2011).

[2] Z. Xie, T. Z. Markus, S. R. Cohen, Z. Vager, R. Gutiérrez, and R. Naaman, Nano Lett. 11, 4652 (2011).

[3] D. Mishra, T. Z. Markus, R. Naaman, M. Kettner, B. Gohler, H. Zacharias, N. Friedman, M. Sheves, and C. Fontanesi, Proc. Nat. Acad. Sci. USA 110, 14872 (2013).
[4] S. Yeganeh, M. A. Ratner, E. Medina, and V. Mujica, J. Chem. Phys. 131, 014707 (2009).

[5] E. Medina, F. López, M. A. Ratner, and V. Mujica, Europhys. Lett. 99, 17006 (2012).

[6] R. Gutiérrez, E. Díaz, R. Naaman, and G. Cuniberti, Phys. Rev. B 85, 081404 (2012).

[7] R. Gutierrez, E. Díaz, C. Gaul, T. Brumme, F. DomínguezAdame, and G. Cuniberti, J. Phys. Chem. C 117, 22276 (2013). 
[8] A. A. Eremko and V. M. Loktev, Phys. Rev. B 88, 165409 (2013).

[9] D. Rai and M. Galperin, J. Phys. Chem. C. 117, 13730 (2013).

[10] E. Medina, L. A. González-Arraga, D. Finkelstein-Shapiro, B. Berche, and V. Mujica, J. Chem. Phys. 142, 194308 (2015).

[11] R. A. Caetano, Sci. Rep. 6, 23452 (2016).

[12] E. Díaz, R. Gutiérrez, C. Gaul, G. Cuniberti, and F. DomínguezAdame, AIMS Mater. Sci. 4, 1052 (2017).

[13] A.-M. Guo and Q.-F. Sun, Phys. Rev. Lett. 108, 218102 (2012).

[14] A.-M. Guo and Q.-F. Sun, Proc. Natl. Acad. Sci. USA 111, 11658 (2014).

[15] S. Matityahu, Y. Utsumi, A. Aharony, O. Entin-Wohlman, and C. A. Balseiro, Phys. Rev. B 93, 075407 (2016).

[16] A.-M. Guo, E. Díaz, C. Gaul, R. Gutierrez, F. DomínguezAdame, G. Cuniberti, and Q.-F. Sun, Phys. Rev. B 89, 205434 (2014).

[17] H.-N. Wu, X. Wang, and W.-J. Gong, Chem. Phys. Lett. 677, 131 (2017).

[18] K. Michaeli, V. Varade, R. Naaman, and D. H. Waldeck, J. Phys.: Condens. Mat. 29, 103002 (2017).

[19] N. A. Zimbovskaya, Transport Properties of Molecular Junctions (Springer, New York, 2013).
[20] S. Fratini, D. Mayou, and S. Ciuchi, Adv. Funct. Mater. 26, 2292 (2016).

[21] P. Albares, E. Díaz, J. M. Cerveró, F. Domínguez-Adame, E. Diez, and P. G. Estévez, Phys. Rev. E 97, 022210 (2018).

[22] E. Díaz, P. Albares, P. G. Estévez, J. M. Cerveró, C. Gaul, E. Diez, and F. Domínguez-Adame, N. J. Phys. 20, 043055 (2018).

[23] S. Behnia, S. Fathizadeh, and A. Akhshani, J. Phys. Chem. C 120, 2973 (2016).

[24] S. V. Salazar, V. Mujica, and E. Medina, CHIMIA International Journal for Chemistry 72, 411 (2018).

[25] D. Hennig and G. P. Tsironis, Phys. Rep. 307, 333 (1999).

[26] W. G. J. Hol, Prog. Biophys. Mol. Biol. 45, 149 (1985).

[27] R. Naaman and D. H. Waldeck, J. Phys. Chem. Lett. 3, 2178 (2012).

[28] O. Bang, P. L. Christiansen, F. If, K. O. Rasmussen, and Y. B. Gaididei, Phys. Rev. E 49, 4627 (1994).

[29] A. S. Davydov, Phys. Scripta 20, 387 (1979).

[30] K. Mallory and R. A. Van Gorder, Phys. Rev. E 89, 013204 (2014).

[31] L. Covaci and M. Berciu, Phys. Rev. Lett. 102, 186403 (2009). 